\title{
Pulmonary Haemosiderosis Secondary to Hereditary Haemochromatosis; a Case Report
}

\author{
Waqas Jehangir ${ }^{1}$, Alexander D. Karabachev ${ }^{2}$, Elvira R. Umyarova ${ }^{1}$ \\ ${ }^{1}$ Department of Hematology and Oncology, University of Vermont Medical Center, Burlington, \\ Vermont, United States, ${ }^{2}$ Department of Medicine, University of Vermont, Larner College of Medicine, \\ Burlington, Vermont, United States
}

Received: 01 September 2019/Accepted: 29 November 2019

\section{Open aCCess \\ Correspondence: Waqas Jehangir, Department of Hematology and Oncology, University of Vermont Medical Center, 111 Colchester Ave, Burlington, Vermont 17822, United States. \\ Email: wjehangir@hotmail.com \\ Citation: Jehangir W, Karabachev AD, Umyarova ER Pulmonary haemosiderosis secondary to hereditary haemochromatosis; a case report. J Cancer Allied Spec [Internet]. 2020 Jan.6; 6(1):e1002957. https://doi. org/10.37029/jcas.v6i1.281}

Copyright: (c) 2020 Jehangir, et al. This is an open access article distributed under the terms of the Creative Commons Attribution License, which permits unrestricted use, distribution, and reproduction in any medium, provided the original author and source are credited.

Funding: Nill.

Competing interests: Nill.

\section{Introduction}

Hereditary haemochromatosis $(\mathrm{HH})$ is an autosomal recessive condition of dysregulated iron absorption that may lead to an overload of total body iron with associated secondary tissue damage. The genetic prevalence of this disorder is $0.4 \%$ in Northern Europeans; however, it has a much lower clinical prevalence. ${ }^{[1]}$ Individuals with a homozygous substitution of tyrosine for cysteine at position 282

\begin{abstract}
Introduction: Hereditary haemochromatosis $(\mathrm{HH})$ is an autosomal recessive disease of increased intestinal absorption of iron, leading to accumulation in tissues which may progress to organ damage, most commonly in the liver. Iron deposition in the liver can lead to cirrhosis and hepatocellular carcinoma. Other common manifestations of haemochromatosis include diabetes, bronzing of the skin, arthropathy and cardiomyopathy. Here, we describe a case of pulmonary haemosiderosis secondary to $\mathrm{HH}$. Case Description: A 49-year-old male with no medical history or family history of iron overload presented with fatigue, shortness of breath and chest pain after a recent finding of elevated ferritin. The patient was found to have biallelic $\mathrm{C} 282 \mathrm{Y}$ mutations of the human homeostatic iron regulator protein (HFE) protein and after further workup with laboratory tests and imaging was diagnosed with $\mathrm{HH}$ with secondary pulmonary haemosiderosis. The patient is receiving twice weekly phlebotomies and has had an overall improvement in his symptoms. Practical Implications: The presentation of haemochromatosis can vary widely depending on the severity of iron overload and the presence of conditions that predispose organ dysfunction. Pulmonary haemosiderosis is a very rare manifestation of $\mathrm{HH}$. This report illustrates the various manifestations of this disease and provides insight into this rare presentation to improve the diagnosis of this disease.
\end{abstract}

Key words: Dyspnoea, ferritins, haemochromatosis protein, haemochromatosis, haemosiderosis, mutation
(C282Y) in the HFE protein account for $82-90 \%$ of the clinical diagnosis of $\mathrm{HH}$ among Northern Europeans descendants. ${ }^{[2]}$ Elevated serum ferritin and transferrin saturation (TSAT) occur more frequently in males with C282Y homozygosity than females with the same amino acid substitution. ${ }^{[3-5]}$ European Association for the Study of the Liver defines $\mathrm{HH}$ as $\mathrm{C282Y}$ homozygosity and increased body iron stores with or without clinical symptoms. ${ }^{[1]}$ 
Iron accumulation associated with $\mathrm{HH}$ may present in a variety of ways. Elevated serum alanine aminotransferase levels indicating that hepatic dysfunction is an early manifestation of this disease and may progress to cirrhosis and hepatocellular carcinoma. ${ }^{[6,7]}$ Iron may accumulate in the skin, leading to pigmentation as well as in endocrine organs resulting in hypothyroidism, diabetes mellitus and hypogonadism. ${ }^{[8,9]}$ In a severely iron overloaded state, cardiomyopathy may occur resulting in arrhythmias and heart failure. ${ }^{[10]} \mathrm{Here}$, we describe $\mathrm{HH}$ presenting with pulmonary haemosiderosis which is a rare presenting manifestation of this disease.

\section{Case Report}

A 49-year-old White male with no significant medical history or family history of iron overload was referred to haematology oncology for further evaluation of an elevated ferritin. The patient had not seen a medical provider for many years before a recent visit to an urgent care clinic for fatigue and left-sided face and left extremity paresthesia. On further workup including possible Vitamin B12 deficiency, he was found to have elevated haemoglobin of $17.6 \mathrm{~g} / \mathrm{dl}$, a ferritin level above $1300 \mathrm{ng} / \mathrm{mL}$ with a normal Vitamin B12 level, white blood cell and platelet counts. A referral to haematology oncology department was placed.

Four weeks after the urgent care visit, the patient presented to the emergency room with episodes of shortness of breath and chest pain. His chest X-ray showed fine reticular nodular pattern throughout the entirety of both lungs concerning for pulmonary haemosiderosis [Figure 1]. His electrolytes and liver function test were within normal limits. His workup showed normal Vitamin B12 levels and an elevated ferritin level of $1913 \mathrm{ng} / \mathrm{mL}$. He denied any major issues or problems. On further workup at the haematology clinic, he specifically denied any history of diabetes, chest tenderness, joint pain, shortness of breath, cough, haemoptysis, history of prior lung disease, frequent respiratory illnesses, fever, chills, night sweats and gastrooesophageal reflux disease. Before diagnosis, he was going to the gym regularly using the treadmill and light weights without limitation. He endorsed having fatigue and numbness and tingling in his hands and feet. He denied any other joint arthritis or darkening of the skin. He had no family history of iron overload and had no offspring. He was a former smoker and quit 1 week before the visit. He denied any alcohol consumption. His physical examination was completely benign.

The blood haemochromatosis human homeostatic iron regulator protein (HFE) gene analysis showed

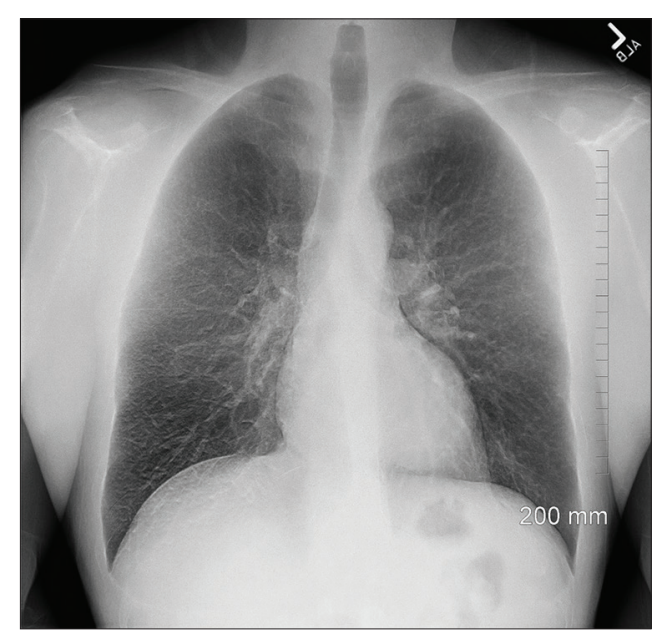

Figure 1: Chest $X$-ray showing fine reticular nodular pattern throughout the entirety of both lungs concerning for pulmonary haemosiderosis

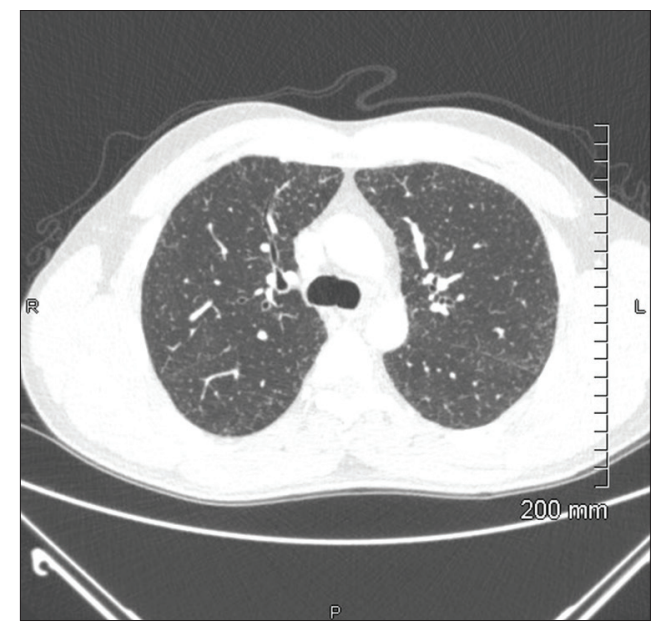

Figure 2: Computed tomography chest showing diffuse reticular nodular opacities consistent with pulmonary haemosiderosis 
two copies of the $\mathrm{C} 282 \mathrm{Y}$ mutation. This result can take place in patients with $\mathrm{HH}$. However, it does not confirm a diagnosis or predisposition to the development of $\mathrm{HH}$. Computed tomography (CT) chest showed diffuse reticular nodular opacities which are consistent with pulmonary haemosiderosis ${ }^{[11]}[$ [Figure 2]. Likewise, the patient underwent FerriScan (Resonance Health, Burswood, Western Australia, Australia) which showed no focal abnormality on non-contrast sequences and marked T2 hypointensity of the liver which is consistent with iron deposition. FerriScan transverse relaxation rate shows that TSAT was $>45 \%$ and his liver iron content

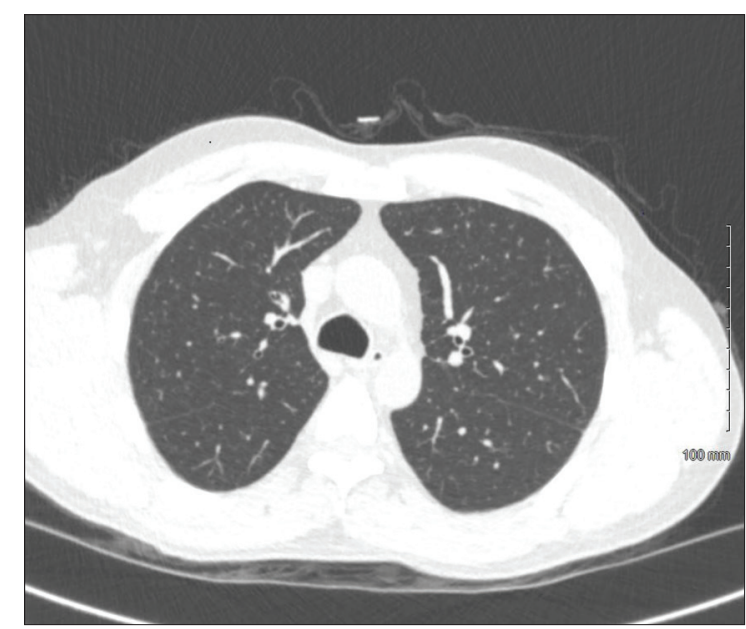

Figure 3: Computed tomography (CT) chest performed 5 months after initial CT scan with minimal residual abnormality with a reported improvement in diffuse reticular nodular opacities per dry liver weight was approximately $29 \mathrm{mg} / \mathrm{g}$ (normal is $<1.8 \mathrm{mg} / \mathrm{g}$ ) [Figure 3].

\section{Diagnosis and Management}

Differential included interstitial lung disease due to his smoking history as well as pulmonary haemosiderosis. Given the elevated ferritin, two copies of the $\mathrm{C} 282 \mathrm{Y}$ mutation and the imaging results, the patient was diagnosed with $\mathrm{HH}$ with secondary pulmonary haemosiderosis. He was started on a weekly phlebotomy schedule for the goal ferritin of $50 \mathrm{ng} / \mathrm{mL}$, while holding phlebotomy if haematocrit reached 33\%. During follow-up, a laboratory test showed a ferritin of $1223 \mathrm{ng} / \mathrm{mL}$ and haemoglobin of $13.4 \mathrm{~g} / \mathrm{dl}$. The patient was placed on twice weekly phlebotomy $(500 \mathrm{ml})$ with oral fluid replacement and a course of prednisone $40 \mathrm{mg}$ daily for 2 weeks, tapered down to $30 \mathrm{mg}$ for 2 weeks and then to $20 \mathrm{mg}$ daily, as well as a prophylaxis sulfamethoxazole/trimethoprim (800/160 mg tablet) every alternate day, 3 days a week. At 3 -month follow-up, he reported an improvement in his fatigue. A repeat chest $C T$ was conducted after 5 months of prior CT [Figure 4] and it showed minimal residual abnormality with a reported improvement in diffuse reticular nodular opacities.

\section{Discussion}

The presentation of $\mathrm{HH}$ can vary depending on the severity of iron overload, the organ

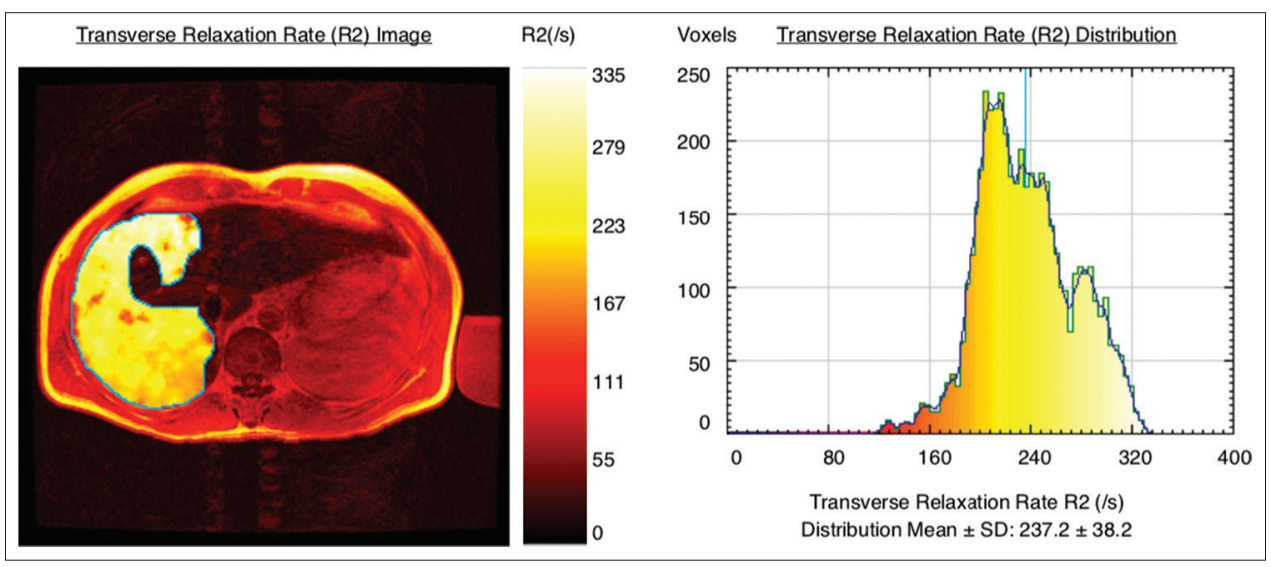

Figure 4: Ferri Scan shows that the transverse relaxation rate transferrin saturation was $>45 \%$ and his liver iron content per dry liver weight was approximately $29 \mathrm{mg} / \mathrm{g}$ (normal is $<1.8 \mathrm{mg} / \mathrm{g}$ ) 
involved and the presence of other conditions that may lead to organ dysfunction. Before our understanding of the genetics of $\mathrm{HH}$ and the HFE gene, the majority of patients presented with symptoms caused by iron accumulation. Analysis of a population of patients from 1959 to 1992 with haemochromatosis concluded that $75 \%$ of individuals presented with abnormalities in liver function tests, $74 \%$ of patients had weakness and lethargy, $70 \%$ of patients presented with hyperpigmentation, $48 \%$ of patients had concurrent diabetes mellitus, $44 \%$ of patients had arthralgia, $31 \%$ of patients presented with electrocardiogram abnormalities and $45 \%$ of male patients had impotence at the time of presentation. ${ }^{[12]}$ While there is a wide array of possible manifestations of $\mathrm{HH}$, pulmonary haemosiderosis is rarely reported.

At present, routine availability of iron studies and genetic testing has led to earlier diagnosis and less severe progression of the disease. A more recent study found that $76 \%$ of individuals who were diagnosed with $\mathrm{HH}$ were asymptomatic at the time of testing. ${ }^{[13]}$ Pulmonary haemosiderosis is not a common presenting sign of $\mathrm{HH}$ and is more often a result of recurrent episodes of diffuse alveolar haemorrhage. This may be due to infection, rheumatic disease, medications or inhalation exposures; however, the aetiology of pulmonary haemosiderosis is often unknown. ${ }^{[14]}$

Pulmonary haemosiderosis secondary to haemochromatosis has been reported once previously in the Spanish medical literature in Archivos de Bronconeumologia in 1995. ${ }^{[15]}$ In this case report, authors performed a transbronchial biopsy, which confirmed iron deposits in alveolar macrophages. Pathognomic feature of pulmonary haemosiderosis is lung biopsy, which should be suggestive for the presence of haemosiderin-laden macrophages. However, this was no conducted in the present case report due to genetic evidence of $\mathrm{HH}$, the imaging findings and the overall improvement in symptoms following repeated therapeutic phlebotomy.
Here, we report a case of a 49-year-old male with an elevated ferritin level who presented with shortness of breath and chest pain. His symptoms were likely due to haemosiderosis secondary to $\mathrm{HH}$ which was confirmed with iron studies, genetic testing and FerriScan magnetic resonance imaging. With this report, we hope to illuminate a rare possible manifestation of $\mathrm{HH}$ and encourage further research to assess if more patients with $\mathrm{HH}$ have pulmonary findings.

\section{Conflicts of Interest}

The authors declare that they have no conflicts of interest.

\section{References}

1. Pietrangelo A, Deugnier Y. EASL clinical practice guidelines for HFE hemochromatosis. J Hepatol 2007;53:3-22.

2. Adams P, Barton J. Haemochromatosis. Lancet 2007;370:1855-60.

3. Olynyk J, Cullen D. A population-based study of the clinical expression of the hemochromatosis gene. N Engl J Med 1999;341:1708-8.

4. Andersen R. Hemochromatosis mutations in the general population: Iron overload progression rate. Blood 2004;103:2914-9.

5. Delatycki M, Allen K, Nisselle A, Collins V, Metcalfe S, du Sart D, et al. Use of community genetic screening to prevent HFE-associated hereditary haemochromatosis. Lancet 2005;366:314-6.

6. Kowdley K. Iron, hemochromatosis, and hepatocellular carcinoma. Gastroenterology 2004; 127:S79-86.

7. Crownover B, Covey C. Hereditary Hemochromatosis; 2019. Available from: https://www.aafp.org/ afp/2013/0201/p183.html. [Last accessed on 2019 Sep 01].

8. McNeil L, McKee L, Lorber D, Rabin D. The endocrine manifestations of hemochromatosis. Am J Med Sci 1983;285:7-13.

9. Utzschneider K, Kowdley K. Hereditary hemochromatosis and diabetes mellitus: Implications for clinical practice. Nat Rev Endocrinol 2010;6:26-33.

10. Gulati V, Harikrishnan P, Palaniswamy C, Aronow W, Jain D, Frishman W. Cardiac involvement in hemochromatosis. Cardiol Rev 2014;22:56-68.

11. Kamienska E, Urasinski T, Gawlikowska-Sroka A, Glura B, Pogorzelski A. Idiopathic pulmonary hemosiderosis in a 9-year-old girl. Eur J Med Res 2009;14 Suppl 4:112-5. 
12. Niederau C, Strohmeyer G, StremmelW. Epidemiology, clinical spectrum and prognosis of hemochromatosis. Adv Exp Med Bio 1994;356:293-302.

13. Cherfane C, Hollenbeck R, Go J, Brown K. Hereditary hemochromatosis: Missed diagnosis or misdiagnosis? Am J Med 2013;126:1010-5.

14. Ioachimescu O, Kotch A, Stoller J. Idiopathic pulmonary hemosiderosis in adults. Clin Pulm Med 2005;12:16-25.

15. Pereiro A, Garcia G, Muniz M, Campa A. Pulmonary interstitial disease due to hemochromatosis as a presenting sign of disease. Arch Bronconeumol 1995;31:9.

\section{Authorship Contributions}

Conceived and designed the analysis: WJ, ADK, ERU; Collected the data: N/A; Performed the analysis: N/A; Wrote the paper: WJ, ADK, ERU. 